\title{
Amphiphilic Polyelectrolytes with Various Hydrophobic Groups: Intramolecular Hydrophobic Aggregation in Aqueous Solution
}

\author{
Yotaro MORISHIMA, Takaomi KOBAYASHI, and Shun-ichi NOZAKURA \\ Department of Macromolecular Science, Faculty of Science, Osaka University, \\ Toyonaka, Osaka 560, Japan
}

(Received July 29, 1988)

\begin{abstract}
To study the effect of the structural factors of hydrophobic residues in amphiphilic polyelectrolytes on self-aggregation and on the nature of hydrophobic microdomains thus formed in aqueous solution, copolymers of sodium 2-acrylamido-2-methylpropanesulfonate (AMPS) with various hydrophobic monomers such as 1-vinylpyrene, 9-vinylphenanthrene, cholestanyl methacrylate, and lauryl methacrylamide were synthesized. The microphase separation of these copolymers to form micelle-like hydrophobic domains in aqueous solution was evidenced by viscosity behavior, the line broadening.of ${ }^{1} \mathrm{H}$ NMR absorption peaks, the surface tension of the aqueous solutions, and the morphology of casi films. The copolymers with aromatic hydrophobic residues (pyrenyl or phenanthryl groups) were more able to self-aggregate than those with aliphatic hydrophobic residues (lauryl or cholestanyl groups). In the hydrophobic microdomains the aromatic residues tend to be more tightly packed than are the aliphatic residues.

KEY WORDS Amphiphilic Polyelectrolytes / Hydrophobic Aggregation /

Microphase Structure / Structural Organization / Hydrophobic

Microdomain / Morphology / Surface Activity /
\end{abstract}

In recent years, there has been much attention focused on photophysics and photochemistry, ${ }^{1-5}$ redox processes, ${ }^{6}$ and chemical reactions $^{7.8}$ in amphiphilic polyelectrolytes in aqueous media because significant kinetic effects are often observed as a result of the existence of the microphase structures of the polymers.

On an amphiphilic polyelectrolyte chian attractive hydrophobic interactions among hydrophobic residues compete with repulsive electrostatic interactions among charged segments. The self-organization of hydrophobic residues to form a micelle-like microphase structure can occur when the hydrophobic interactions are strong enough to prevail over segmental Coulombic repulsions. Naturally, the consequence of the competition between the hydrophobic attraction and the electrostatic repulsion strongly depends on the con- tent of the hydrophobic residues in the polymers. $^{9}$

We have reported that amphiphilic copolymers of AMPS with 9-vinylphenanthrene (9VPh) show anomalous photophysical behavior in aqueous solution as compared with a low molecular-weight related model system. ${ }^{10}$ In these amphiphilic copolymers the clusters of phenanthrene (Phen) residues are formed, to which hydrophobic quencher molecules are efficiently bound in aqueous solution. ${ }^{10.11}$

More recently, we have demonstrated a remarkable effect of the "hydrophobic compartmentalization" of chromophores (Phen residues) in the hydrophobic microdomains composed of pendant phenyl groups or lauryl groups in the amphiphilic terpolymers of AMPS, 9VPh, and styrene or lauryl methacrylamide (LMA) ${ }^{12}$ In the case of the compartmentalization with the phenyl aggregates, 
a collisionless long-range electron transfer occurs from singlet-excited Phen to methylviologen $\left(\mathrm{MV}^{2+}\right)$ bound to the peripheral area of the hydrophobic microdomain; i.e., $\mathrm{MV}^{2+}$ is prohibited from entering the phenyl aggregates. ${ }^{13}$ By contrast, in the case of the lauryl aggregates, $\mathrm{MV}^{2+}$ can easily get into the hydrophobic microdomain and is allowed to come into contact with the Phen residues, and thereby ground-state interactions between Phen and $\mathrm{MV}^{2+}$ are favored. A remarkable feature for the hydrophobic compartmentalization of the chromophores with the phenyl aggregates is that an extremely efficient charge separation takes place. ${ }^{12,13}$

These observations in our continuing work on photophysics and photoinduced electron transfer in amphiphilic polyelectrolyte systems have motivated us to study how the tendency for the microphase separation and the nature of the microphase structures thus formed are dependent on the type of the hydrophobic groups.

To this end we prepared random copolymers of AMPS with various hydrophobic monomers (Scheme 1). The present paper describes the microphase separation of amphiphilic polyelectrolytes in aqueous solution with an emphasis on the qualitative comparison among the various types of hydrophobic groups in the copolymers. The experimental results obtained in the present study are not satisfactorily quantitative ones. Nevertheless, the qualitative comparison among the various hydrophobic residues can still provide useful information on the characteristic photophysical and photochemical behavior of the amphiphilic polyelectrolytes in aqueous solution.

\section{EXPERIMENTAL}

\section{Materials}

Lauryl methacrylamide (LMA) was synthesized as follows: To a stirred solution of $35.2 \mathrm{~g}$ $(0.19 \mathrm{~mol})$ of laurylamine (Wako Pure Chem-
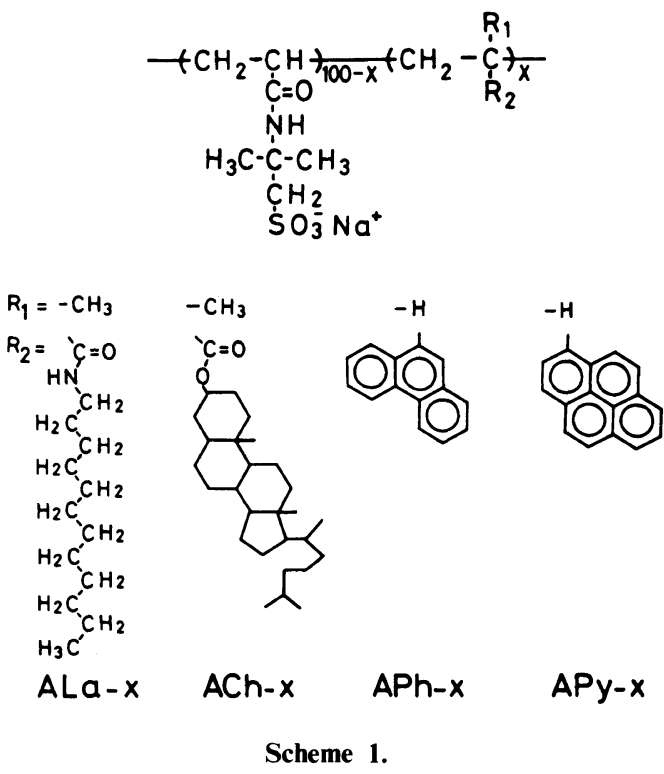

ical Ind. Ltd. $), 35.5 \mathrm{~g}(0.35 \mathrm{~mol})$ of triethylamine, and $10 \mathrm{mg}$ of hydroquinone in $400 \mathrm{ml}$ of benzene was added $20.3 \mathrm{~g}(0.194 \mathrm{~mol})$ of methacryloyl chloride over a period of $1 \mathrm{~h}$. The reaction mixture was stirred for additional $24 \mathrm{~h}$ at room temperature. After removing precipitates by filtration the benzene solution was washed with a $0.1-\mathrm{M} \mathrm{HCl}$ aqueous solution three times and then thoroughly washed with pure water. The organic layer was then dried over magnesium sulfate overnight. Benzene was removed on a rotary evaporator to dryness. The crude product was recrystallized twice from benzene and dried in vacuo at room temperature $\left(36.8 \mathrm{~g}\right.$, yield $76.4 \%$ ): $\mathrm{mp} 36^{\circ} \mathrm{C} ;{ }^{1} \mathrm{H}$ NMR $\left(\mathrm{CDCl}_{3}\right) 1.8-1.91(\mathrm{t}, 3 \mathrm{H}), 2.24-2.6$ $(\mathrm{m}, 20 \mathrm{H}), 2.94(\mathrm{~m}, 3 \mathrm{H}), 4.18-4.38(\mathrm{q}, 2 \mathrm{H})$, $6.28(\mathrm{~m}, 1 \mathrm{H}), 6.64(\mathrm{~s}, 1 \mathrm{H})$, and $6.9(\mathrm{~s}, 1 \mathrm{H})$; Anal. Calcd. for $\mathrm{C}_{16} \mathrm{H}_{31} \mathrm{NO}: \mathrm{C}, 75.83 \% ; \mathrm{H}$, $12.33 \%$; N, $5.52 \%$. Found: C, $75.59 \% \mathrm{H}$, $12.38 \%$; N , $5.51 \%$.

The preparations of cholestanyl methacrylate $^{14}$ (ChM), 9-Vinylphenanthrene ${ }^{10}$ (9VPh), and 1 -vinylpyrene ${ }^{10}$ (1VPy) have been reported elsewhere.

2-Acrylamido-2-methylpropanesulfonic acid (AMPS) (Nitto Chemical Co.) was used 
without further purification.

\section{Copolymerizations}

Copolymers of AMPS with the hydrophobic monomers were prepared by free-radical polymerization as follows: In a glass ampule were placed a known amount of AMPS and a hydrophobic monomer, $0.2 \mathrm{~mol} \%$ (on the basis of the total monomers) of 2,2'-azobis (2-methylpropionitrile) (AIBN), and tetrahydrofuran (THF). The complete dissolution of AMPS in THF was effected by adding the equimolar triethylamine to the solution. The ampule was thoroughly outgassed on a vacuum line and sealed off. The polymerizations were carried out in the ampule immersed in a water bath at $60^{\circ} \mathrm{C}$. The reaction mixture was then poured into a large excess of $n$-hexane to precipitate out the resultant copolymers. The copolymers were further purified by reprecipitation from a THF solution into an excess of $n$-hexane, allowed to air dry briefly, and treated with dilute aqueous sodium hydroxide. With water soluble copolymers, the dilute alkaline solution was shaken with $n$-hexane to extract liberated triethylamine and dialyzed against pure water. The copolymers were recovered by freeze-drying. With water insoluble copolymers, the precipitates in the dilute alkaline solution were filtered, washed with pure water, and dried.

The copolymer compositions were determined by the $\mathrm{C} / \mathrm{N}$ and $\mathrm{C} / \mathrm{S}$ ratios of microanalysis.

\section{Measurements}

The reduced viscosities of the aqueous solutions of the copolymers were measured at $30^{\circ} \mathrm{C}$ in a modified Ubbelohde type viscometer.

${ }^{1} \mathrm{H}$ NMR spectra were measured employing 6-wt $\%$ solutions of the copolymers in DMSO$d_{6}$ or in $\mathrm{D}_{2} \mathrm{O}$ at room temperature by using a Varian T-60 spectrometer.

Surface tensions were measured by a conventional drop-weight method. The number of drops was counted at $30^{\circ} \mathrm{C}$ by a stalagnometer placed in a thermostat. ${ }^{15}$

Electron micrographs were taken with a Hitachi H-600FE Transmission Analytical Electron Microscope at $100 \mathrm{kV}$. An aqueous solution of the amphiphilic copolymer ( $c a .2$ $\mathrm{wt}^{\mathrm{o}} \%$ ) was applied to a 400-mesh copper grid covered with a carbon-coated collodion film and allowed air dry. The cast film was stained by applying a drop of $2-\mathrm{wt} \%$ aqueous solution of uranyl acetate. The specimens were observed at the direct magnification of $5 \times 10^{4}$.

\section{RESULTS AND DISCUSSION}

The segments of AMPS in the amphiphilic copolymers can effectively solubilize the sequences of hydrophobic monomer units into water. For the amphiphilic copolymers with lauryl methacrylamide (LMA) $\left(\mathrm{C}_{16} \mathrm{H}_{31} \mathrm{NO}\right)$, ALa- $x(x=4,13$, or 44$)$, all the copolymers .were soluble in water, while the copolymers with cholestanyl methacrylate $(\mathrm{ChM}) \quad\left(\mathrm{C}_{31}\right.$ $\left.\mathrm{H}_{52} \mathrm{O}_{2}\right)$, ACh- $x(x=1,9,23$, or 60$)$, were less soluble; i.e., ACh-23 (ACh- $x$ with $x=23$ ) was mostly soluble in water, whereas ACh-60 was completely insoluble. This difference in solubility in water between ALa- $x$ and ACh- $x$ seems to be reasonable because the ChM units are considerably more hydrophobic than the LMA units in terms of the number of carbon atoms contained in the monomer. On the other hand, the copolymers of 9-vinylphenanthrene (9VPh) $\left(\mathrm{C}_{16} \mathrm{H}_{12}\right)$, APh- $x(x=2,5,19,41$, or 50 ), and of 1-vinylpyrene (1 VPy) $\left(\mathrm{C}_{18} \mathrm{H}_{12}\right)$, $\operatorname{APy}-x(x=3,10$, or 50$)$, were all soluble in water. These four types of the copolymers prepared in the present study were all soluble in methanol, $\mathrm{N}, \mathrm{N}$-dimethylformamide, and dimethylsulfoxide, but insoluble in most of other common organic solvents.

Figure 1 shows the plots of the reduced viscosities of various amphiphilic polyelectrolytes with varying copolymer compositions $(x)$ as a function of the concentration of the copolymer in salt-free aqueous solution. 


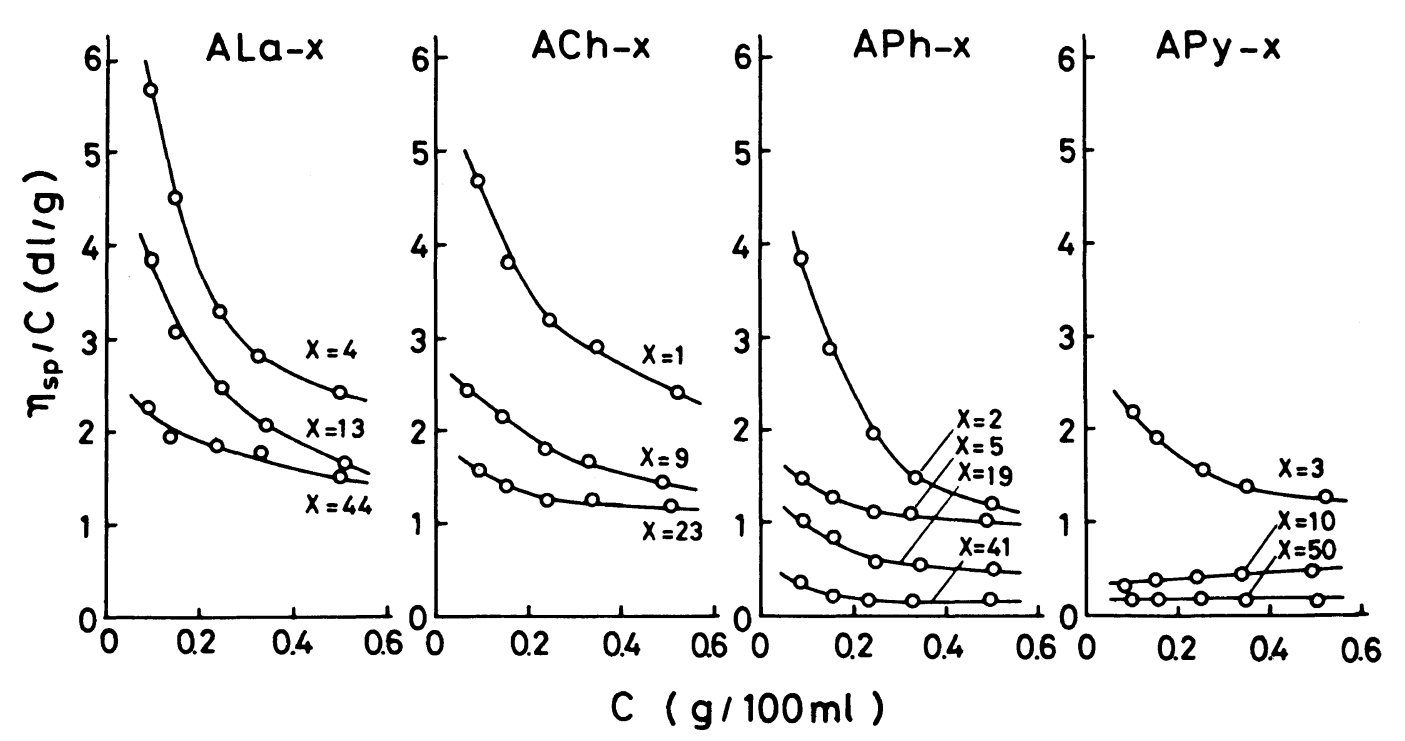

Figure 1. Plots of reduced viscosities $\left(\eta_{\mathrm{sp}} / c\right) v s$. concentration for the various amphiphilic polyelectrolytes with varying copolymer compositions in pure water at $30^{\circ} \mathrm{C}$.

Copolymers with a low content of hydrophobic units showed a negatively sloping plot, a typical behavior of ionized polyelectrolytes. The steepness of the negative slopes reflects the extent of chain expansion on dilution, which is caused by an increased segmental electrostatic repulsion. ${ }^{16}$ If intramolecular hydrophobic interactions are strong enough not to allow a polymer coil to expand, the reduced viscosity would not increase on dilution. In fact the negative slope tends to get less steep as the content of the hydrophobic units in the copolymes increases. In the case of APy- $x$ the negative slope turned into a slightly positive slope and the reduced viscosities significantly decreased as the content of the pyrene residues was increased from $x=3$ to 10 . This is an implication that in the case of the pyrene residues intramolecular aggregations among the hydrophobic groups are highly favored and the pyrene aggregates already exist when $x=10$. From relatively less steep slopes observed for APh- $x$ and APy- $x$ it is suggested that the amphiphilic copolymers with the aromatic hydrophobic groups have a marked tendency to exist in a compact conformation in dilute aqueous solution as compared to the copolymers with aliphatic hydrophobic groups (ALa- $x$ and ACh- $x$ ).

Viscosity is not only related to molecular weight but also reflects the extent of intramolecular hydrophobic association of hydrophobic residues on which the hydrodynamic volume of the polymer coils would strongly depened. The reduced viscosities for the copolymers with aliphatic hydrophobic units in pure water are significantly large as compared with those for the copolymers with aromatic hydrophobic units. It should be noted here, however, that the difference in the viscosities between the aromatic and aliphatic types of the amphiphilic copolymers becomes much less when a salt is added. For the aromatic copolymers a decrease in the reduced viscosity upon addition of a salt is significantly small as compared to the aliphatic copolymer case (data not shown). This suggests that the aromatic copolymers are shrunk owing to "preexisting" hydrophobic self-aggregation of the aromatic units even under salt-free conditions. Namely, the chain expansion in saltfree water is much more difficult in the case of 

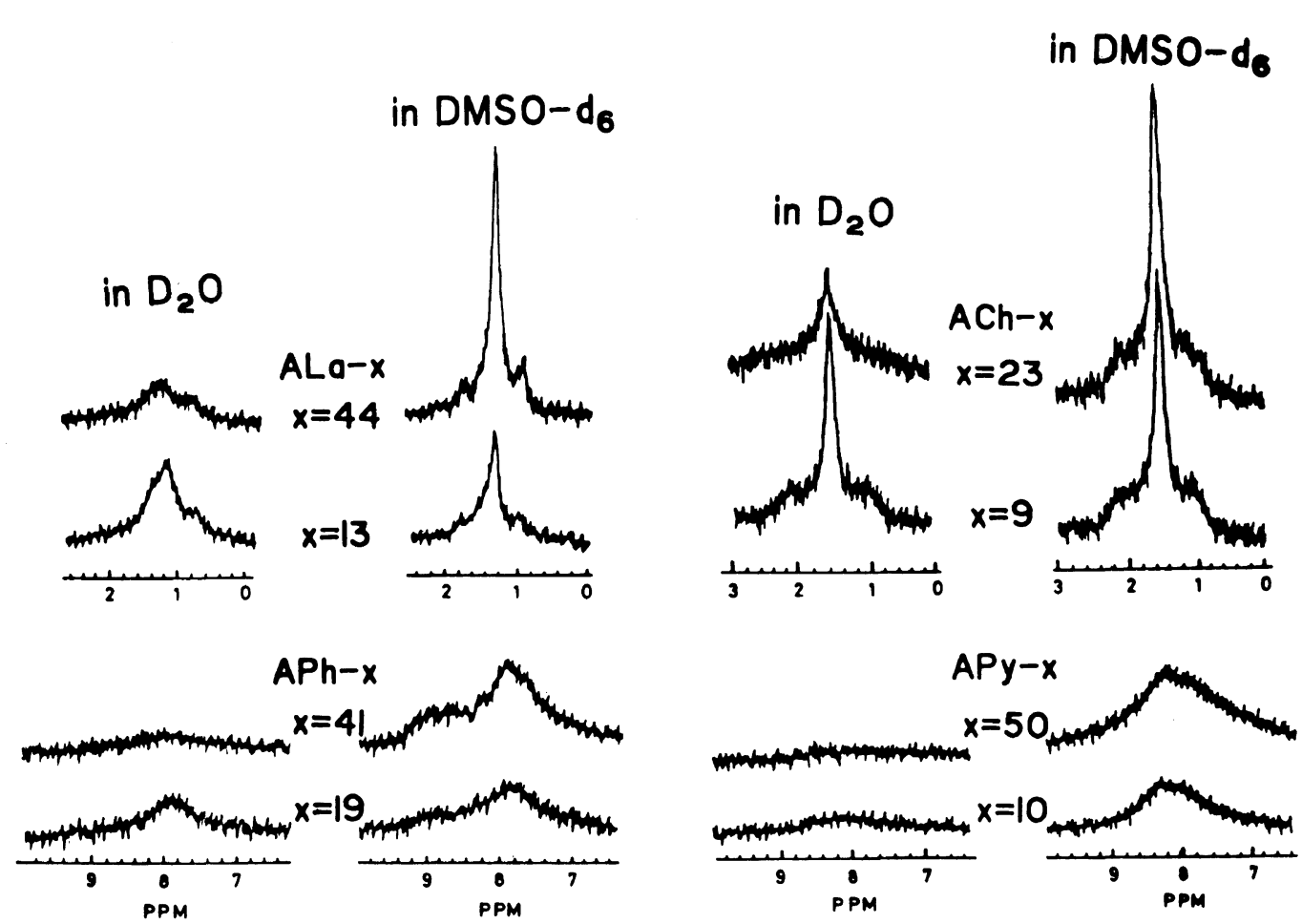

Figure 2. ${ }^{1} \mathrm{H}$ NMR spectra of amphiphilic polyelectrolytes with various copolymer compositions in $\mathrm{D}_{2} \mathrm{O}$ and in DMSO- $d_{6}$ : The concentration of sample solutions, $6 \mathrm{wt} \%$; temperature, $25^{\circ} \mathrm{C}$.

the copolymers with aromatic hydrophobic units.

Figure 2 shows the ${ }^{1} \mathrm{H}$ NMR absorption peaks associated with methyl and methylene protons for ALa- $x$ and ACh- $x$ and with ring protons for APh- $x$ and APy- $x$. These spectra were measured in $\mathrm{D}_{2} \mathrm{O}$ and in DMSO- $d_{6}$ at the same polymer concentration $(6 \mathrm{wt} \%)$. In DMSO- $d_{6}$ the intensities for these peaks increased systematically with an increase in the $\mathrm{mol} \%$ content of the hydrophobic units in the copolymers. On the other hand, in $\mathrm{D}_{2} \mathrm{O}$ the peaks showed an extensive broadening with increasing $x$ value due to the self-aggregation of the hydrophobic groups occurring in aqueous solution. This line broadening is more pronounced for the aromatic hydrophobic units than for the aliphatic ones. This suggests that polycyclic aromatic rings tend to be tightly packed when hydrophobically selfaggregate in aqueous solution, whereas ali- phatic groups, regardless of whether they are flexible chains (lauryl groups in $\mathrm{ALa}-x$ ) or rigid rings (cholestanyl groups in $\mathrm{ACh}-x$ ), tend to self-associate rather loosely.

The formation of hydrophobic microdomains is exhibited by surface-active effects as illustrated in Figure 3. The surface tensions of the aqueous solutions of the amphiphilic copolymers decreased with an increase in the polymer concentration. The surface-active effect is more significant for copolymers with larger $x$ and is eminent for the copolymers with aromatic hydrophobic units as compared to the aliphatic copolymers. Furthermore, the pyrene copolymers showed a larger surfaceactive effect than did the phenanthrene copolymers (compare APh-19 and APy-10 in Figure 3). Inflection points observed for the plots in Figure 3 correspond to the critical concentrations at which intermolecular cooperative associations through hydrophobic 


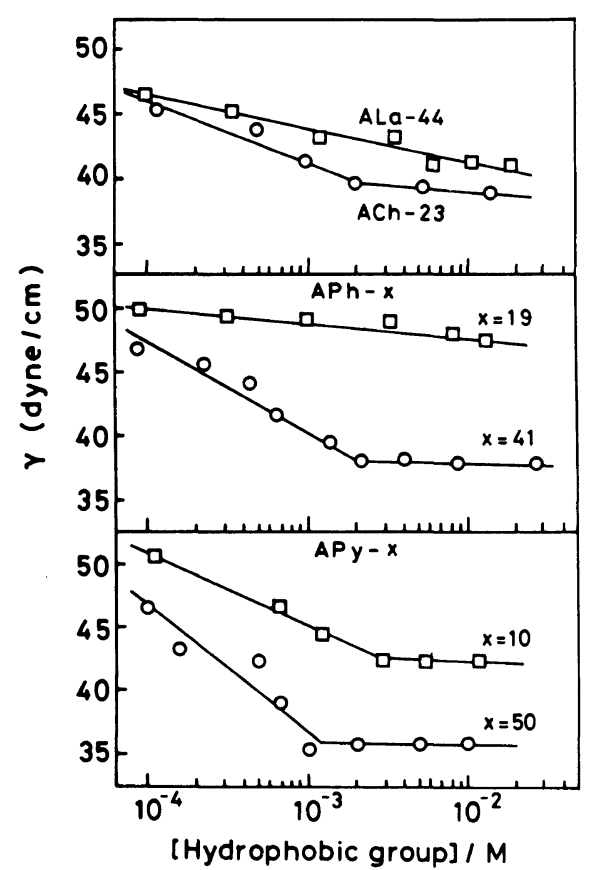

Figure 3. Relationship between surface tension and the concentration of hydrophobic residues in the amphiphilic polyelectrolytes in aqueous solution at $30^{\circ} \mathrm{C}$.

interactions begin to occur among the amphiphilic copolymers. There seems little or no such cooperative intermolecular association processes for the copolymers with aliphatic hydrophobic groups.

Figure 4 compares transmission electron micrographs (TEM) of the cast films of the amphiphilic copolymers stained with uranyl acetate. In all cases microphase structures are clearly observed (unstained white portions are due to hydrophobic microdomains). It is to be noted that, if specimen films are prepared by casting from organic solutions of the amphiphilic copolymers, no such microphase structures are observed in the TEM. As can be seen from Figure 4 there is a significant difference in the type of microphase separation to form secondary structures among the kinds of the hydrophobic units in the amphiphilic copolymers. There also seems to be differences in the tertiary or higher-order structures due to an extensive aggregation of the secondary structures. The shapes of the hydrophobic microdomains for the aliphatic hydrophobic residues are quite different from those for the aromatic hydrophobic residues. In the former case, thread-like hydrophopobic domains were observed. In particular, with ALa-44, long thread-like domains with a thickness of roughly $20 \mathrm{~A}$ can be clearly seen in the picture. With ACh-23, the length and the thickness of the hydrophobic domains are much smaller than those with ALa-44. For the aromatic hydrophobic groups, both APh-50 and APy-50 seem to undergo coagulation to result in somewhat spherical domains. Although we cannot interpret in detail the morphology of the amphiphilic polyelectrolyte films at the present time, it is noteworthy that different types of the hydrophobic groups would give considerably different morphological features. The morphologies observed in the TEM do not necessarily represent those in aqueous solution, but, it is, at least, conceivable that similar microphase separation is already present in aqueous solution.

Hydrophobic associations are characterized by the release of "ice-like" structured water molecules around hydrophobic groups into free water in the bulk phase, thus resulting in an increase in entropy. ${ }^{17}$ Therefore, the hydrophobic residues tend to associate with one another so as to attain the smallest possible surface area exposed to the aqueous phase to release the largest possible amount of the structured water. Accordingly, "sterical-fit" between the hydrophobic groups may be an important factor for this process. A possible explanation for the tighter hydrophobic interactions between the aromatic hydrophobic groups may be that planar aromatic rings can sterically fit in with each other favorably to release a large amount of structured water as compared to the case of the aliphatic hydrophobic groups.

Besides the chemical structures of the hydrophobic groups, the distribution of the hydrophobic sequences in the copolymers may 

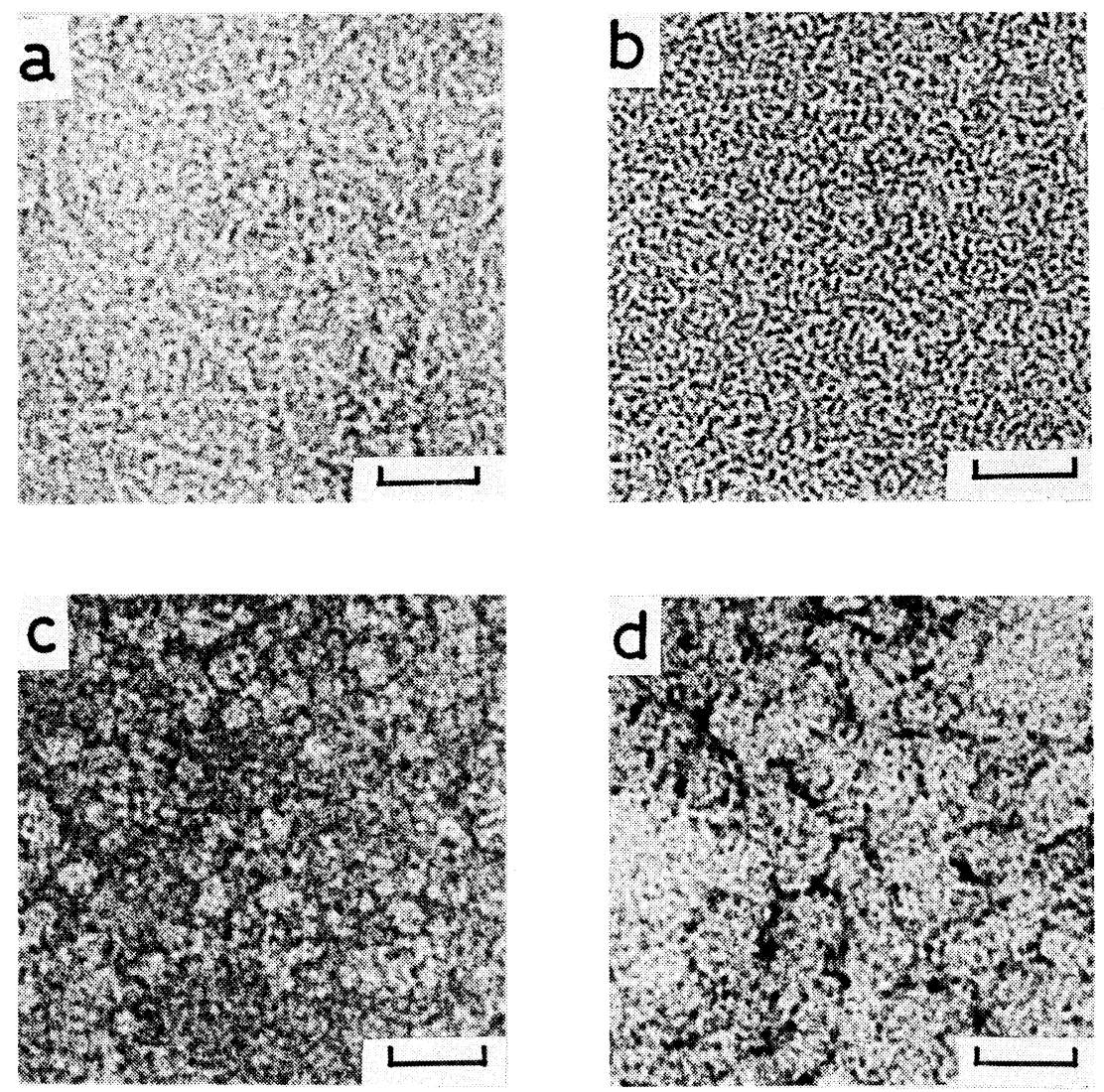

Figure 4. Transmission electron photomicrographs of films cast from the aqueous solutions of the amphiphilic polyelectrolytes: a, ALa-44; b, ACh-23; c, APlh-41; d, APy-50. Scale: $20 \mathrm{~nm}$.

have an influence on the amphiphilic nature of the copolymers.

Work is in progress to clarify this point by preparing the copolymers of AMPS with methacrylamides having various hydrophobic substituent groups. In these copolymers the hydrophobic units are expected to be distributed in the completely random fashion.

Acknowledgements. This work was supported in part by a Grant-in-Aid for Scientific Research (No. 59430019) from the Ministry of Education, Science, and Culture of Japan. The authors are grateful to Dr. A. Koreeda and Mr. T. Ishibashi of Osaka University for the measurements of the electron microscope.

\section{REFERENCES}

1. (a) Y. Morishima, Y. Itoh, T. Hashimoto, and S. Nozakura, J. Polym. Sci., Polym. Chem. Ed., 20, 2007 (1982). (b) Y. Morishima, T. Tanaka, Y. Itoh, and S. Nozakura, Polym. J., 14, 861 (1982). (c) Y. Morishima, Y. Itoh, and S. Nozakura, Chem. Phys. Lett., 91, 258 (1982). (d) Y. Morishima, Y. Itoh, S. Nozakura, T. Ohno, and S. Kato, Macromolecules, 17, 2264 (1984). (e) Y. Itoh, Y. Morishima, and S. Nozakura, Photochem. Photobiol., 39, 451, 603 (1984). (f) Y. Morishima, T. Kobayashi, S. Nozakura, and S. E. Webber, Macromolecules, 20, 807 (1987).

2. (a) J. E. Guillet, Y. Takahashi, A. R. McIntosh, and J. R. Bolton, Macromolecules, 18, 1788 (1985). (b) J. 
E. Guillet and W. A. Rendall, ibid., 19, 224 (1986). (c) J. E. Guillet, J. Wang, and L. Gu, ibid., 19, 2793 (1986).

3. (a) J. A. Delaire, M. A. J. Rodgers, and S. E. Webber, J. Phys. Chem., 88, 6219 (1984). (b) F. Bai, C.-H. Chang, and S. E. Webber, Macromolecules, 19, 588, 2488, 2798 (1986). (c) S. E. Webber, ibid., 19, 1658 (1986). (d) J. A. Delaire, M. A. J. Rodgers, and S. E. Webber, Eur. Polym. J., 22, 189 (1986).

4. (a) N. J. Turro and K. S. Arora, Polymer, 27, 783 (1986). (b) K. S. Arora, K.-C. Hwang, and N. J. Turro, Macromolecules, 19, 2806 (1986). (c) K. S. Arora and N. J. Turro, J. Polym. Sci., Polym. Phys. Ed., 25, 243 (1987). (d) K. S. Arora and N. J. Turro, ibid., 25, 259 (1987).

5. (a) D.-Y. Chu and J. K. Thomas, Macromolecules, 17, 2142 (1984). (b) R. Harrop, P. A. Williams, and J. K. Thomas, J. Chem. Soc., Chem. Commun., 280 (1985).

6. (a) F. C. Anson, J.-M. Saveant, and K. Shigehara, J. Am. Chem. Soc., 105, 1096 (1983). (b) F. C. Anson, T. Ohsaka, and J.-M. Saveant, J. Phys. Chem., 87, 640 (1983). (c) D. D. Montgomery and F. C. Anson, J. Am. Chem. Soc., 107, 3431 (1985).

7. N. Sbiti and C. Tondre, Macromolecules, 17, 369 (1984).
8. J. Jager and J. B. F. N. Engberts, J. Org. Chem., 50, 1474 (1985).

9. Y. Morishima, Y. Itoh, and S. Nozakura, Makromol. Chem., 182, 3135 (1981).

10. Y. Itoh, Y. Morishima, and S. Nozakura, J. Polym. Sci., Polym. Chem. Ed., 20, 467 (1982).

11. Y. Morishima, T. Kobayashi, and S. Nozakura, $J$. Phys. Chem., 89, 4081 (1985).

12. Y. Morishima, T. Kobayashi, T. Furui, and S. Nozakura, Macromolecules, 20, 1707 (1987).

13. Y. Morishima, T. Furui, S. Nozakura, T. Okada, and N. Mataga, J. Phys. Chem., in press.

14. A. C. de Visser, K. de Groot, J. Feyen, and A. Bantjes, J. Polym. Sci., Polym. Lett. Ed., 10, 851 (1972).

15. (a) A. E. Alexander and J. B. Hayter, In "Technique of Chemistry, Physical Methods of Chemistry," Part V, A. Weissberg and B. Bryant, Ed., WileyInterscience, New York, N.Y., 1971, p 512. (b) K. Tsuji and T. Takagi, Protein, Nucleic Acid, Enzyme, 19, 705 (1974).

16. H. Morawetz, "Macromolecules in Solution," 2nd ed., Interscience, New York, N.Y., 1975, p 359.

17. W. P. Jencks, "Catalysis in Chemistry and Enzymology," McGraw-Hill, New York, N.Y., 1969, p 393. 